\title{
Onset of convection of a reacting fluid layer in a porous medium with temperature-dependent heat source
}

\author{
C. Israel-Cookey ${ }^{1,{ }^{*}}$, V. B. Omubo-Pepple ${ }^{2}$ \\ ${ }^{1}$ Department of Mathematics, Rivers State University of Science and Technology Port \\ Harcourt. *Corresponding author, Email: Israel-cookey.chigozie@ust.edu.ng \\ ${ }^{2}$ Department of Physics, Rivers State University of Science and Technology Port Harcourt
}

\begin{abstract}
This paper investigates the problem of double-diffusive convection in a horizontal layer filled with a reacting fluid with temperature-dependent internal heat source within the Darcy limit. The linear stability theory is applied for the onset of both stationary (monotonic) and oscillatory convection. The critical Rayleigh numbers for the onset of convection are determined in terms of the governing parameters. The results show that positive increments in the internal heat source parameter hasten the onset on both stationary and oscillatory convection.
\end{abstract}

\section{INTRODUCTION}

The study of the onset of thermosolutal or double diffusive convection in fluid saturated porous layer has been an active area of research interest for many years. These phenomena of combined heat and mass transfers where both temperature and solute fields contribute to the buoyancy of the fluid have many applications in the behaviour of fluids in the crust of the earth, geophysics, metallurgy, material science and petroleum engineering. For instance, in geological processes thermosolutal convection in porous media may be important in dolomitisation of carbonate platforms (Kaufman 1994), soil salinisation (Gilman and Bear 1994) and heat transfer in geothermal reservoirs (Oldenburg and Pruess 1988). Comprehensive reviews of the literature on double diffusive natural convection in porous media and its applications can be found in Nield and Bejan (2006).

The earliest investigations of the effects of chemical reactions on the stability of a fluid layer were carried out by Wollkind and Frisch (1971a, b), who considered the problem of the stability with the effect of dissociation. Bdzil and Frisch (1971) investigated the same problem but considered the effects of catalysis at the lower boundary of the layer. Steinberg and Brand (1984) investigated the effect of chemical reaction in a porous medium. In this study they considered the regime where the chemical reaction was sufficiently fast that the solutal diffusion could be neglected. More recently, D'Hernoncourt et. al. $(2006,2007)$ considered the effect of exothermic reaction on double - diffusive convection in a fluid saturated porous medium; while Hill (2005) considered the problem of linear and nonlinear convection in a fluid saturated porous medium with a concentration based internal heat source. Pritchard and Richardson (2007) investigated the problem of the onset of thermosolutal convection of a binary fluid in a horizontal porous layer with temperature dependent solubility using linear stability analysis.

The aim of this present paper is to investigate the effect of temperature-dependent internal heat source on the onset of thermosolutal convection in a horizontal layer filled with a reacting fluid in the Darcy limit.

Mathematical Formulation: We consider a first order chemically reacting fluid layer of height $h>0$, heated and salted from below and bounded between two impermeable horizontal surfaces located at $z^{\prime}=-\frac{h}{2}$ and $z^{\prime}=\frac{h}{2}$. The lower and upper surfaces are maintained at temperatures $T_{1}$ and $T_{2}$ and solutal mass concentrations $c_{1}$ and $c_{2}$, respectively. The fluid is assumed to be Newtonian with constant physical properties except for the density, $\rho$ in the buoyancy term, which according to Boussinesq approximation depends on the temperature, $T^{\prime}$ and specie concentration, $c^{\prime}$ as follows

$\rho\left(T^{\theta}{ }_{0} c^{v}\right)=\rho_{0}\left[1-\beta_{T}\left(T^{v}-T_{0}\right)+\beta_{c}\left(c^{\theta}-c_{0}\right)\right]$

where $\beta_{T}, \beta_{c}$ are the thermal and solutal expansion coefficients, respectively, $\rho_{0}$ is the reference 
density. Also, $T_{0}=\frac{T_{1}+T_{2}}{2}, T_{1}>T_{2} \quad$ and $c_{0}=\frac{c_{1}+c_{2}}{2}, c_{1}>c_{2}$.

Further, the internal heat source is modelled linearly with respect to temperature. This is represented by the introduction of the term $Q_{0}\left(T^{\prime}-T_{0}\right)$ in the energy equation, where $Q_{0}$ is some constant of proportionality. We take a horizontal coordinate $x^{\prime}$ and a vertical coordinate $z^{\prime}$ which is increasing upwards. Making use of Darcy law and Boussinesq approximation, the appropriate governing equations are (Nield and Bejan (2006))

$$
\begin{aligned}
& \boldsymbol{\nabla} \cdot \mathbf{V}^{\prime}=0 \\
& \boldsymbol{\nabla}^{\prime} P^{\prime}+\frac{\mu}{K} \boldsymbol{V}^{\prime}+\rho\left(T^{\prime}, c^{\prime}\right) g \boldsymbol{k}=0 \\
& \left(\rho c_{p}\right)_{\mathrm{m}} \frac{\partial Y^{v}}{\partial t^{y}}+\left(\rho c_{p}\right)_{f} V^{0} \cdot \nabla T^{0}=k_{\mathrm{m}} \nabla^{2} T^{v}+Q_{0}\left(T^{v}-T_{0}\right) \\
& \phi \frac{\partial c^{\prime}}{\partial t^{\prime}}+\boldsymbol{V} \cdot \boldsymbol{\nabla} c^{\prime}=D_{m} \boldsymbol{\nabla}^{2} c^{\prime}-k_{r}\left(c^{\prime}-c_{0}\right)
\end{aligned}
$$

where $g$ is the acceleration due to gravity, $\boldsymbol{k}$ is a unit vector in the $z^{\prime}$ direction, $\boldsymbol{V}$ is the Darcy velocity, $P^{\prime}$ is the pressure, $K$ is the permeability of the porous medium, $\phi$ is the porosity of the porous medium, $k_{m}$ is the thermal diffusivity of the porous medium and $D_{m}$ is the solutal diffusivity of the medium. The subscripts $m$ and $f$ denotes the medium and the fluid respectively.

The boundary conditions are

$$
\begin{array}{ll}
W^{\prime}=0, T^{\prime}=T_{1}, c^{\prime}=c_{1} & \text { on } z^{\prime}=-\frac{h}{2} \\
W^{\prime}=0, T^{\prime}=T_{2}, c^{\prime}=c_{2} & \text { on } z^{\prime}=\frac{h}{2}
\end{array}
$$

We non-dimensionalize Equations (2) - (6) by introducing the following dimensionless variables

$$
\begin{aligned}
& (x, y, z)=\frac{1}{h}\left(x^{\prime}, y^{\prime}, z^{\prime}\right) ; \quad t=\frac{\alpha_{m} t y}{A h^{2}} ; \quad V=\frac{h V t}{\alpha_{m}} ; \\
& P=\frac{K}{\mu \alpha_{m}}\left(P^{\prime}+\rho_{0} g z^{\prime}\right) ; A=\frac{\left(\rho c_{p}\right)_{m}}{\left(\rho c_{p}\right)_{f}} ; \\
& \alpha_{m}=\frac{k_{m}}{\left(\rho c_{p}\right)_{f}} ; T=\frac{T^{\prime}-T_{0}}{T_{1}-T_{2}} ; c=\frac{c^{t}-c_{0}}{c_{1}-c_{2}}
\end{aligned}
$$

The dimensionless equations are

$$
\boldsymbol{\nabla} \cdot \mathbf{V}=0
$$

$$
\begin{gathered}
\boldsymbol{V}=-\nabla P+\left(R_{a} T-R_{e} c\right) k \\
\frac{\partial T}{\partial t}+\boldsymbol{V} \cdot \boldsymbol{\nabla} T=\left(\nabla^{2}+Q\right) T \\
\phi \frac{\partial c}{\partial t}+\boldsymbol{V} \cdot \boldsymbol{\nabla} c=\frac{1}{L e} \nabla^{2} c-k_{r} c
\end{gathered}
$$

subject to the boundary condition

$$
W=0, T= \pm \frac{1}{2}, c= \pm \frac{1}{2} \text { on } z=\mp \frac{1}{2}
$$

where $R a=\frac{K h_{g} \beta_{T}\left(T_{1}-T_{2}\right)}{\alpha_{m 1} v}$ is the thermal Rayleigh number, $R c=\frac{K h g \beta_{d}\left(c_{1}-\omega_{2}\right)}{\alpha_{m 1} v}$ is the solutal Rayleigh number, $L e=\frac{\alpha_{m}}{D_{m}}$ is the Lewis number, $Q=\frac{h^{2} Q_{0}}{\alpha_{m}\left(\rho c_{p}\right)_{f}}$ is the internal heat source parameter and $k_{r}=\frac{k_{r} h^{2}}{\alpha_{m}}$ is the reaction rate parameter.

\section{Stability Analysis}

Steady State Solutions: We seek an initial steady state solutions for which $\boldsymbol{V}=0$ and $\frac{\partial}{\partial t} \rightarrow 0$. We then find

$$
\begin{aligned}
& \frac{d P_{B}}{d z}=R a T_{B}-R c c_{B} \\
& \frac{d^{2} T_{B}}{d z^{2}}+Q T_{B}=0 \\
& \quad \frac{d^{2} c_{B}}{d z^{2}}-k_{r} \text { Le } c_{B}=0
\end{aligned}
$$

subject to

$$
T_{B}=c_{B}= \pm \frac{1}{2} \quad \text { on } z=\mp \frac{1}{2}
$$

Solving Equations (13) - (15) together with conditions (16) yield the steady state of the systems as

$$
\begin{gathered}
T_{B}=-\frac{1}{2} \frac{\sin [z \alpha]}{\sin \left[\frac{\alpha}{2}\right]}, \alpha=\sqrt{Q} \\
C_{B}=\frac{1}{2} \frac{\operatorname{Sinh}[\mathrm{kz}]}{\operatorname{Sinh}\left[\frac{\pi}{2}\right]}, R=\sqrt{\operatorname{Lek} k_{r}} \\
P_{B}=\frac{R_{a} \operatorname{Cos}[\alpha z]}{2 \alpha \operatorname{Sin}\left[\frac{\alpha}{2}\right]}-\frac{R_{C} \operatorname{Cosh}[\mathrm{kz}]}{2 R \operatorname{Sinh}\left[\frac{R}{2}\right]}
\end{gathered}
$$

\subsection{Linearized Equations and Perturbation Model}

To access the stability of the steady state solutions, we superimpose small perturbations on the basic state in the form [Chandrasekhar (1961); Drazin and Reid (2004); Nield and Bejan (2006)] 
$V=0+v_{s} T=T_{B}+\theta_{s} c=c_{B}+\varphi_{,} P=P_{B}+p(20)$

Substituting (20) into (8) - (12) and neglecting higher order terms of the perturbed quantities, we obtain the linearized perturbed equations

$$
\begin{aligned}
& \boldsymbol{\nabla} \cdot \boldsymbol{v}=0 \\
& \boldsymbol{\nabla} p=(R a \theta-R c \varphi) k-\boldsymbol{v} \\
& \frac{\partial \theta}{\partial t}+\alpha_{1} W=\left(\nabla^{2}+Q\right) \theta \\
& b \frac{\partial \varphi}{\partial t}+\alpha_{2} W=\frac{1}{L \theta}\left(\nabla^{2}-\mathrm{k}_{\mathrm{r}}\right) \varphi
\end{aligned}
$$

where $\alpha_{1}=\frac{\partial T_{B}}{\partial z}$ and $\alpha_{2}=\frac{\partial c_{B}}{\partial z}$ are the temperature and species gradients in the fluid, and $b=\phi$.

Next, we eliminate the pressure perturbations by operating on (22) twice with the curl operator and using the continuity equation (21). Taking only the $z-$ component, the resulting equation becomes

$$
\boldsymbol{\nabla}^{2} w=\operatorname{Ra} \boldsymbol{\nabla}_{h}^{2} \theta-\operatorname{Rc} \boldsymbol{\nabla}_{h}^{2} \varphi
$$

where $\nabla_{h}^{2}=\frac{\partial^{2}}{\partial x^{2}}+\frac{\partial^{2}}{\partial y^{2}}$ is the Laplace operator with respect to the horizontal plane. The boundary conditions are now

$w=0, \theta=0, \varphi=0$ at $z= \pm \frac{1}{2}$

Normal Mode Analysis: To proceed with our analysis, we consider the expansions of the form $(w, \theta, \varphi)=(W(z), \Theta(z), \Psi(z)) \exp (\Omega t)$

where $\Omega=\sigma+i \omega$ is complex, and $\sigma_{y} \omega$ are real numbers. Substituting (27) into (23) - (25) and letting $D=\frac{\partial}{\partial z}$, we obtain the following system of equations

$$
\begin{aligned}
& \left(D^{2}-a^{2}+Q-\Omega\right) \Theta=\alpha_{1} W \\
& \left(D^{2}-a^{2}-L e k_{r}-b \text { Le } \Omega\right) \Psi=\alpha_{2} \text { Le W (29) } \\
& \left(D^{2}-a^{2}\right) \Theta+a^{2} R a \Theta-\mathrm{a}^{2} \operatorname{Rc} \Psi
\end{aligned}
$$

where $a^{2}$ is a wave number arising from the separation of variables and $f(x, y)$ is a horizontal plane form tiling the plane $(x, y)$ periodically and satisfies $\nabla_{h}^{2} f+a^{2} f=0$ (Christopherson (1940), Hill (2005)). The boundary conditions are

$$
\begin{aligned}
W & =0, \Theta=0, \Psi=0 \text { on } z= \pm 1 / 2 \\
D^{2} W & =0 \text { on a free surface. }
\end{aligned}
$$

Next, system (28) - (30) is further reduced to a single scalar equation in $W$ by eliminating $\Theta$ and $\Psi$. The result is

$$
\begin{aligned}
& \left(D^{2}-a^{2}\right)\left(D^{2}-a^{2}+Q-\Omega\right)\left(D^{2}-a^{2}-\mathbb{L} e k_{y}-b \text { Le } \Omega\right) W= \\
& -a_{1}^{2} a^{2} R a\left(D^{2}-a^{2}-L e k_{y}-b \text { Le } \Omega\right) W- \\
& a_{2}^{2} a^{2} R c\left(D^{2}-a^{2}+Q-\Omega\right) W
\end{aligned}
$$

now subject to

$W=D^{2} W=D^{4} W=\cdots=0$

For an idealized fluid layer with free boundaries in which the boundary conditions (33) hold, the solution of (33) is possible if

$$
W(z)=w_{0} \sin [\pi z]
$$

where $w_{0}$ is a constant. Substituting (34) into (32), we obtain the dispersion relation

$$
R a=\frac{1}{\Gamma}\left(\frac{s_{1}\left(s_{2}+\Omega\right)}{a^{2}}+\frac{L e \alpha_{2}\left(s_{2}+\Omega\right)}{s_{\mathrm{a}}+b \operatorname{Le} \Omega}\right)
$$

where

$$
\begin{aligned}
& s_{1}=a^{2}+\pi^{2}, s_{2}=a^{2}+\pi^{2}-Q, s_{1}=a^{2}+\pi^{2}+ \\
& \text { Le } k_{y}, \Gamma=\frac{\operatorname{asin}[\alpha z Z]}{2 \sin \left[\frac{[}{2}\right]}, a=\sqrt{Q}
\end{aligned}
$$

\section{RESULTS AND DISCUSSION}

At marginal stability $\sigma=0$, and so $\Omega=i \omega$. With this (35) reduces to

$$
\begin{aligned}
& R a=\frac{1}{\Gamma}\left(\frac{s_{1} S_{2}+i s_{1} \omega}{a^{2}}+\frac{L e \alpha_{2} R e}{a_{a}^{2}+b^{2} L e^{2} \omega^{2}}\left(s_{2} s_{3}+\right.\right. \\
& \left.b L e \omega^{2}+i \omega\left(s_{3}-b L e s_{2}\right)\right)
\end{aligned}
$$

For stationary convection, we put $\omega=0, R a=R a(s), a=a_{c}$ in (36) and obtain after simplification yields

$$
R a(s)=\frac{1}{\Gamma}\left(\frac{s_{1} s_{2}}{a_{E}^{2}}+\frac{L e \alpha_{2} s_{2}}{s_{\mathbb{M}}} R c\right)
$$


$=\left(\frac{\left(\pi^{2}+a_{e}^{2}\right)\left(\pi^{2}+a_{e}^{2}-Q\right)}{a_{e}^{2}}+\frac{L e \alpha_{2}\left(\pi^{2}+a_{e}^{2}-Q\right)}{\pi^{2}+a_{e}^{2}+L e k_{x}} R c\right)$

Equation (37) represents the boundary for monotonic or stationary instability. In particular, to

find the lowest threshold of instability as a function of the wave number, $a_{c}$ we compute $\frac{\partial R a(s)}{\partial a_{c}^{2}}=0$. This yields the following $8^{\text {th }}$ order polynomial

$a_{e}^{9}+2 b_{2} a_{e}^{6}+\left(b_{2}^{2}+b_{1}+b_{a}\right) a_{e}^{4}+2 b_{1} b_{2} a_{e}^{2}+b_{2}^{2} b_{1}=0$

where

$b_{1}=\pi^{2} Q-\pi^{4}$, $b_{2}=\pi^{2}+L e k_{r}$,

$b_{3}=$ Le $\alpha_{2}\left(\right.$ Le $\left.k_{r}+Q\right) R c$.

In order to compare our results with those in literature, we set $Q=0.01, L e=0.01, k_{r}=0.1$, $R c=1000$ (this value corresponds that of salt water) and solve the characteristic polynomial (38) with the help of "mathematica version 7". This yields $a_{c}=3.14202 \approx \pi$ and we conclude that the critical Rayleigh number for the onset of stationary instability is

$R a(s)_{c r i}=\left(2 \pi^{2}-Q\right)\left(2+\frac{L e R c}{2 \pi^{2}+L e k_{r}}\right)$

If $Q=0=k_{r}$, that is, in the absence of internal heat source and chemical reaction, we obtain the critical Rayleigh number as

$R a(s)_{c r i}=4 \pi^{2}+L e R c$

This is the exact result previously reported by Lambardo et. al. (2003).

To analyse the onset of oscillatory convection for which $\omega \neq 0$, we set $R a=R a(o), a=a_{c}$ and keeping only the imaginary part of (36), we have

$R a(o)=\frac{1}{b L e \Gamma}\left(\beta_{4} a_{c}^{2}+\beta_{2}+\frac{\beta_{0}}{\alpha_{c}^{2}}\right)+\frac{\alpha_{2} R c}{b \Gamma}$

where

$$
\begin{aligned}
& \beta_{0}=\pi^{2}\left(b L e\left(\pi^{2}-Q\right)+\left(\pi^{2}+L e k_{r}\right)\right) ; \\
& \beta_{2}=b L e\left(2 \pi^{2}-Q\right)+\left(2 \pi^{2}+L e k_{r}\right) ; \\
& \beta_{4}=1+b L e
\end{aligned}
$$

By minimizing (40) we obtain the critical Rayleigh number for the onset of overstability (oscillatory instability) as

$$
R a(o)_{c y i}=\frac{1}{b \Gamma}\left(4 \pi^{2}\left(\frac{1+b L q}{b L \theta}\right)+2\left(k_{y}-b Q\right)+\frac{a_{2} A Q}{b}\right)
$$

We remark here that for $\Gamma \rightarrow 1, Q=0, k_{r}=0$ and $\alpha_{2} \rightarrow 1$ in (42), the threshold of oscillatory convection reduces to

$R a(o)_{\text {cri }}=4 \pi^{2}\left(\frac{1+b L e}{b L e}\right)+\frac{R c}{b}$

which is exactly the results of Lambardo et. al.(2003).

Figure 1and 2 depict the graphs of thermal Rayleigh numbers, $R a$ for the onset of both stationary and oscillatory convections plotted against the wave number, $a$, respectively for and varying values of internal heat source parameter, $Q$. The result shows that positive increment in $Q$ cause a reduction in the critical Rayleigh numbers for both stationary and oscillatory convections. This implies that increases in $Q$ hasten the onset of instabilities in the

system.

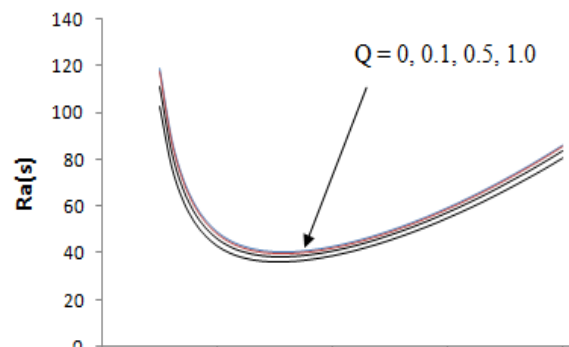

Fig 1. Variation of Rayleigh numbegr, Ra as a function of wave number, $\mathrm{a}$ for $\mathrm{Kr}=0.1, \mathrm{Rc}=$ $1000, b=0.2$, Le $=0.01$ and different values of $Q$

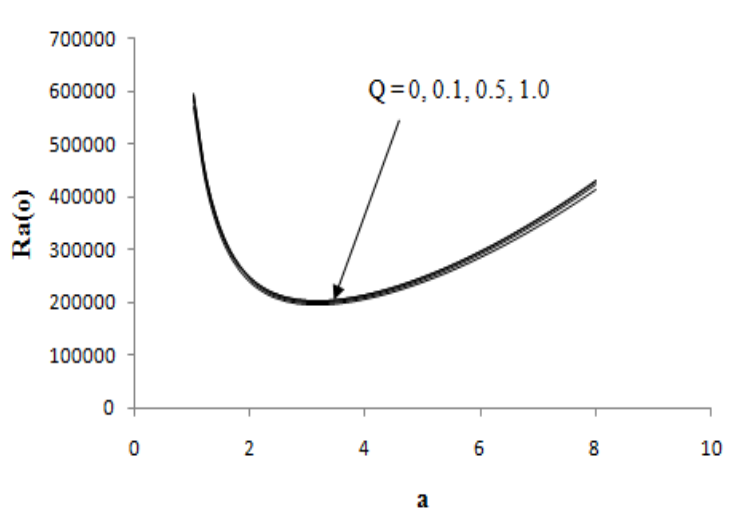

Fig 2. Variation of Rayleigh number, Ra as a function of wave number, a 
In Figures 3 and 4 we depict the graphs of thermal Rayleigh number, $R a$ for the onset of stationary and oscillatory convections plotted against the solutal Rayleigh number, $R c$, respectively for varying values of internal heat source parameter, $Q$. The diagrams show that the effect of increasing the internal heat source parameter allows the onset of instabilities to occur early.

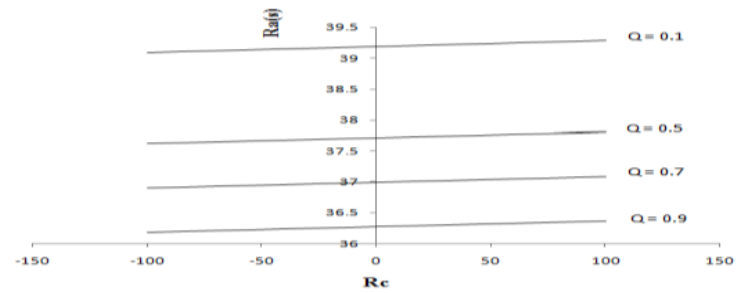

Fig 3. Variation of stationary Rayleigh number, Ra as a function of the solutal Rayleigh number, Rc for $\mathrm{Kr}=0.01, \mathrm{Rc}=1000, \mathrm{~b}=0.2$, Le $=0.01, \mathrm{a}=3$ and different values of $Q$

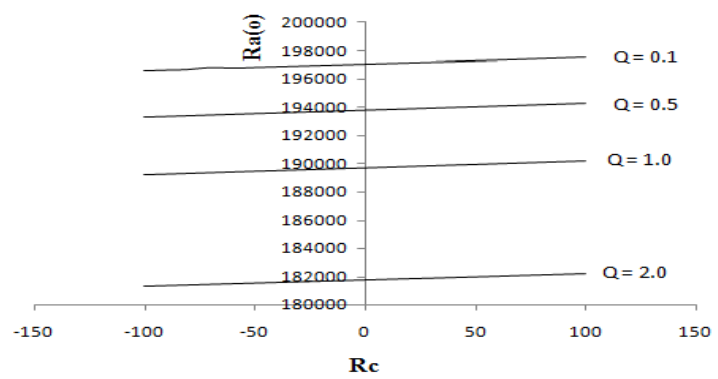

Fig 4. Variation of oscillatory Rayleigh number, $R$ a as a function of wave number, $a$ for $\mathrm{Kr}=0.1, \mathrm{Rc}=1000, \mathrm{~b}=0.2, \mathrm{Le}=0.01$ and different values of $Q$

\section{CONCLUSION}

In this study we used the linear stability analysis to investigate the effect of temperature dependent internal heat source on the onset of stationary (monotonic) and oscillatory instabilities of a first order chemically reacting horizontal fluid layer in a porous medium within the Darcy limit. The results show that positive increments in the internal heat source parameter hasten the onset of both stationary and oscillatory instabilities in the system. That is, increasing values of the internal heat source have a destabilizing effect on the system. Also, in the limiting case when both internal heat source parameter and the reaction parameter are set equal to zero, the results is reduced to known results previous reported in literature on double - diffusive convections.

\section{ACKNOWLEDGEMENT}

The authors are indebted to Mrs. G. Israel - Coookey for excellent typesetting of the manuscript.

\section{REFERENCES}

Bdzil, J., Frisch, H. L.: Chemical instabilities II. Chemical surface reactions and hydrodynamic instability. Phys. Fluids 14 (3) (1971), 475 - 482.

Chandrasekhar, S.: Hydrodynamic and hydromagnetic stability. Oxford University Press, Oxford. 1961.

D’Hernoncourt, J. D., Zebb, A., De Wit, A.: Reaction driven convection around a stably stratified fronts. Phys. Rev. Letters PRL 96 (2006), 154501.

D'Hernoncourt, J. D., Zebb, A., De Wit, A.: Double diffusive instabilities of autocatalytic chemical fronts. J. Fluid Mech. 576 (2007), 445 - 456.

Drazin,P. G., Reid, W. H.: Hydrodynamic stability. Cambridge University Press, Cambridge. 2004.

Gilman, A., Bear, J.: The influence of free convection on soil salinisation in arid regions. Transport in Porous Media 23 (1994), 275 - 301.

Hill, A. A.: Double - diffusive convection in a porous medium with concentration based internal heat source. Proc. R. Soc. A461 (2005), 561 - 574.

Kaufman, J.: Numerical methods of fluid flow in carbonate platforms: implications for dolomitisation. J. Sed. Res. A64, (1994), 128 - 139.

Lombardo, S., Mulone, G., Straughan, B.: Nonlinear stability in the Benard problem for a double - diffusive mixtures in a porous medium. Math. Meth. Applied Sc. 24 (2001), 1229 - 1246.

Neild, D. A., Bejan, A.: Convection in porous media. Springer, New York. 2006Oldenburg, C. M., Pruess, K.: Layered thermohaline convection in hypersaline geothermal systems. Transport in Porous Media 33 (1988), $29-63$.

Pritchard, D., Richardson, C. N.: The effect of temperature - dependent solubility on the onset of thermosolutal convection in a horizontal porous layer. J. Fluid Mech. 571, (2007), 59 - 95.

Steinberg, V., Brand, H.: Convective instabilities of binary mixtures with fast chemical reaction in a porous medium. J. Chem. Phys. 78 (5), (1984), 2655 - 2660.

Wollkind, D. J., Frisch, H. L.: Chemical instability I. A heated horizontal layer of dissociating fluid. Phys. Fluids 14 (1) (1971), $13-18$.

Wollkind, D. J., Frisch, H. L.: Chemical instability III. Nonlinear stability analysis of a heated horizontal layer of dissociating fluid. Phys. Fluids 4(3) (1971), 482 487. 